\title{
EISENIA FETIDA (SAVIGNY, 1826) Y EISENIA ANDREI BOUCHÉ, 1972 SON DOS ESPECIES DIFERENTES DE LOMBRICES DE TIERRA
}

\author{
Jorge Domínguez ${ }^{1}$ \& Marcos PÉrez-Losada ${ }^{2}$ \\ ${ }^{1}$ Departamento de Ecoloxía e Bioloxía Animal. Universidade de Vigo. Vigo E-36310, España. E-mail: \\ jdguez@uvigo.es. \\ ${ }^{2}$ CIBIO, Centro de Investigação em Biodiversidade e Recursos Genéticos Universidade do Porto \\ Campus Agrário de Vairão 4485-661 Vairão, Portugal. E-mail: mlosada@genoma-llc.com
}

Domínguez, J. \& M. Pérez-Losada. 2010. Eisenia fetida (Savigny, 1826) y Eisenia andrei Bouché, 1972 son dos especies diferentes de lombrices de tierra. Acta Zoológica Mexicana (n.s.), Número Especial 2: 321-331.

RESUMEN. Eisenia andrei (Savigny, 1826) y Eisenia fetida Bouché, 1972 (Oligochaeta, Lumbricidae) son dos especies de lombrices de tierra muy relacionadas y utilizadas habitualmente en el proceso de vermicompostaje de residuos orgánicos sólidos, en la ecotoxicología, fisiología y estudios genéticos. Su estatus taxonómico ha sido muy controvertido y en la literatura actual, ambas especies continúan a ser indiscriminadamente referidas como E. fetida o E. foetida; a menudo no queda claro cual de las dos especies ha sido la utilizada. Este artículo de revisión incluye una síntesis de dos trabajos ya publicados por nuestro grupo en los que se demuestra de forma clara que se trata de dos especies biológicas diferentes que están aisladas reproductivamente y que también están bien delimitadas filogenéticamente. El aislamiento reproductivo se confirmó después de estudiar la viabilidad de la descendencia de cruces inter e intraespecíficos de ambas especies de lombrices. Además, los árboles filogenéticos construidos sobre la base de las secuencias de ADN de los genes 28S y COI confirmaron que eran especies filogenéticamente diferentes. El hecho de que sean dos especies diferentes tiene importantes implicaciones; así por ejemplo en los procesos de vermicompostaje es más recomendable la utilización de E. andrei que tiene tasas de crecimiento y reproducción más altas que E. fetida. Por otra parte en estudios de ecotoxicología no es posible asumir que las sustancias contaminantes tendrían el mismo efecto en las dos especies dado que sus respuestas a los factores de estrés podrían ser diferentes. El aislamiento reproductivo de las dos especies es postcópula y no precópula, y esto junto con el hecho de que viven en simpatría afecta claramente a la dinámica de poblaciones reduciendo la eficacia biológica individual da cada especie. Por todo ello es importante considerarlas como dos especies diferentes y evitar los cultivos mixtos.

Palabras clave: Lombrices de tierra, barreras reproductivas, hibridación, especiación, aislamiento postzigótico, DNA, filogenia, delimitación de especies.

Domínguez, J. \& M. Pérez-Losada. 2010. Eisenia fetida (Savigny, 1826) and Eisenia andrei Bouché, 1972 are two different earthworm species. Acta Zoológica Mexicana (n.s.), Número Especial 2: 321-331. 
Domínguez \& Pérez-Losada et al.: Eisenia andrei y Eisenia fetida son dos especies distintas

\begin{abstract}
The closely related species Eisenia fetida (Savigny, 1826) and Eisenia andrei Bouché, 1972 are the most commonly used for management of organic wastes, and also in ecotoxicology, physiology and genetic studies. The problem of their taxonomic status remained unresolved for a long time and in much of the current literature both species are still termed indiscriminately as E. fetida or E. foetida, and it is often not clear which of the two species is being referred to. This review article synthesizes two articles already published by our group where we found that they are two different biological species, reproductively isolated and that they are also two different phylogenetic species. The reproductive isolation was confirmed after studying the offspring viability from inter- and intra-specific crosses of both species. Additionally, fully resolved and well supported phylogenetic trees based on mitochondrial (COI) and nuclear DNA sequences (28S) confirmed that they are different phylogenetic species. This evidence implies important considerations: for vermiculture or vermicomposting $E$. andrei is more recommended since its growth and reproduction rates are higher. In studies on ecotoxicology it is not possible to assume that contaminants will have the same effect on the two species, since their responses to stress factors could be different. The existence of post-copula but not pre-copula isolation in sympatric populations clearly affects the population dynamics by reducing the fitness of the individuals. For this reason, in applied aspects it is important keep the two species separated.
\end{abstract}

Keywords: Earthworms, reproductive barriers, hybridization, speciation, post-zygotic isolation, DNA, phylogeny, species delimitation.

\title{
INTRODUCCIÓN
}

Este trabajo representa una síntesis de dos trabajos ya publicados por nuestro grupo de investigación (Domínguez et al. 2005, Pérez-Losada et al. 2005) en el que se demuestra de forma clara que Eisenia fetida (Savigny, 1826) y Eisenia andrei Bouché, 1972 (Oligochaeta, Lumbricidae) son dos especies biológicas diferentes, reproductivamente aisladas y bien delimitadas filogenéticamente.

La importancia de la taxonomía está bien reconocida por la mayoría de los científicos y de hecho, sin una taxonomía fiable, la mayoría de los estudios ecológicos son irrelevantes. En muchas especies de lombrices de tierra la identificación taxonómica basada en caracteres morfológicos es difícil debido a la ausencia de caracteres diagnósticos estables y fáciles de manejar (Pop et al. 2003).

Eisenia fetida y E. andrei son dos especies de lombrices estrechamente relacionadas y muy utilizadas para el reciclaje de residuos orgánicos mediante vermicompostaje, y también en estudios de ecotoxicología, fisiología y genética. Este uso tan extendido se debe a que son ubicuas con una distribución cosmopolita, con ciclos de vida cortos, un rango amplio de tolerancia a la temperatura y a la humedad y un manejo relativamente sencillo (Domínguez 2004).

Ambas especies se describieron como morfotipos diferentes de $E$. fetida de acuerdo a diferencias en la pigmentación del cuerpo (André 1963) y posteriormente Bouché (1972) les dio estatus subespecífico, nombrándolas E. foetida foetida y E. foetida unicolor. Aunque actualmente muchos autores aceptan a E. fetida y E. andrei como especies diferentes, la literatura más antigua e incluso mucha literatura actual se refiere a estas especies colectivamente como E. fetida o E. foetida, una corrección incorrecta 
e ilegal del original E. fetida (Sims 1983, Easton 1983). Eisenia fetida se corresponde con la forma rayada y presenta el área entre los segmentos sin pigmentación o de color amarillo o pálido; de ahí, su nombre común de lombriz rayada o lombriz tigre. En contraste, E. andrei, la lombriz roja común, tiene la forma de color rojo uniforme. Aparte de las diferencias en la pigmentación, ambas especies son morfológicamente similares (Sims \& Gerard 1985, Reinecke \& Viljoen 1991) y sus parámetros biológicos, sobre todo de potencial reproductivo y sus ciclos de vida no se diferencian significativamente, aunque la tasa de crecimiento y producción de capullos es algo más alta en $E$. andrei que en E. fetida (Elvira et al. 1996). Los ciclos de vida de $E$. fetida y E. andrei y su biología de poblaciones y ecología han sido investigados por varios autores y han sido resumidos por Domínguez (2004). El problema del estatus taxonómico del complejo E. fetida/andrei permanece aun sin resolver y además, en gran parte de la literatura actual, ambas especies son nombradas indiscriminadamente como E. fetida, no quedando claro en muchas ocasiones a cual de las dos especies se hace referencia.

Ambas especies son sintópicas y generalmente viven en colonias mixtas en pilas de estiércol y restos vegetales y por tanto su hibridación sería posible; la hibridación entre poblaciones o especies puede tener efectos perjudiciales en la eficacia biológica y afectar de forma dramática a la dinámica de poblaciones en colonias mixtas. Si eso fuese así, cabría esperar que existiese aislamiento reproductivo que podría ser prezigótico, es decir debido a una incompatibilidad reproductiva en la cópula o postzigótico, es decir debido a una reducción en la viabilidad de los descendientes.

El objetivo de esta investigación fue determinar si E. andrei y E. fetida son dos especies biológica y filogenéticamente diferentes. Se presentan en primer lugar los resultados de algunos experimentos de laboratorio para probar la existencia de barreras reproductivas pre- o postzigóticas comparando la producción de capullos y recién nacidos de las dos especies mediante cruces interespecíficos e intraespecíficos. Además, se presentan los resultados obtenidos utilizando datos moleculares basados en árboles filogenéticos con secuencias de ADN mitocondrial y nuclear y aplicando el procedimiento de delimitación filogenética de especies de Wiens \& Penkrot (2002) para inferir las barreras especificas entre varias poblaciones de E. fetida y E. andrei.

\section{MATERIAL Y MÉTODOS}

Para estudiar el aislamiento reproductivo se utilizaron 4 poblaciones diferentes de lombrices: una población de E. fetida de Vigo (Noroeste de España) y tres poblaciones de $E$. andrei provenientes de Vigo, Madrid y Brasil. Se utilizaron juveniles (100$150 \mathrm{mg}$ peso fresco), que se mantuvieron aislados en placas petri con estiércol de vaca como alimento, para asegurar que las lombrices no almacenaban esperma de cópulas previas. Las placas se mantuvieron en cámaras a $20{ }^{\circ} \mathrm{C}$ y $90 \%$ de humedad. Cuando 
las lombrices alcanzaron la madurez sexual se realizaron cruces entre individuos de las distintas poblaciones. Los individuos se asignaron al azar en los cruces y el peso de los miembros de la pareja en cada cruce fue similar. Cada pareja se colocó en una placa petri durante siete días. Después de este periodo, las lombrices se volvieron a colocar individualmente en sus placa petri originales y durante 15 semanas se registró la producción semanal de capullos, el tiempo de incubación de los mismos, su tasa de viabilidad y el número de recién nacidos por capullo. Se utilizaron modelos lineales generalizados (MLG; Wedderburn 1974, McCullagh \& Nelder 1989) para determinar diferencias significativas en los parámetros reproductivos entre los distintos cruces.

Para el estudio de delimitación filogenética se utilizaron 20 individuos de $E$. andrei procedentes de poblaciones de Brasil, Irlanda y España (cuatro poblaciones en total), y 11 individuos de E. fetida de Irlanda y España (tres poblaciones). Además se utilizaron 6 individuos de E. eiseni (Levinsen, 1884) de España (dos poblaciones) como grupo externo.

El ADN genómico se extrajo usando los métodos descritos por Crandall \& Fitzpatrick (1996). Los productos de PCR para la subunidad 28S del gen ribosomal nuclear y el gen mitocondrial citocromo oxidasa I (COI) se amplificaron usando los primers de Whiting (2001; rd1a a rd6b) y Folmer et al. (1994) respectivamente. Las secuencias se generaron en ambas direcciones en un secuenciador ABI usando el ABI-Big Dye Ready-Reaction Kit. Las secuencias de nucleótidos se alinearon usando Clustal $\mathrm{X}$ (Thompson et al. 1997). La incongruencia entre 28S y COI se determinó mediante la metodología propuesta por Wiens (1998). Se realizaron análisis filogenéticos separados para cada gen con el fin de detectar posibles áreas de incongruencia (donde pueden fallar los análisis filogenéticos combinados) indicadas por nodos conflictivos (soportados por proporciones bootstrap $\geq 70 \%$ o probabilidades posteriores $\geq 95 \%$ ) en los distintos árboles. La selección del modelo evolutivo utilizado en los análisis de máxima verosimilitud y en la aproximación bayesiana se realizó en el programa MODELTEST 3.06 (Posada \& Crandall 1998). La reconstrucción filogenética se realizó con PAUP v4.0b10 (Swofford 2002) utilizando métodos de máxima parsimonia (MP) y máxima verosimilitud (ML, sus siglas en inglés). Los análisis de MP se llevaron a cabo con pesos iguales, 100 adiciones aleatorias de secuencias y permutación global de ramas (TBR, sus siglas en inglés) para buscar el mejor árbol. Para los árboles obtenidos se evaluó la veracidad de los nodos usando la prueba de bootstrap no paramétrico (Felsenstein 1985) con 1000 réplicas y una adición por réplica. La implementación de ML se realizó con 10 adiciones aleatorias de secuencias, TBR y bootstrap no paramétrico con 100 réplicas y una adición por réplica. La aproximación bayesiana se estimó usando MrBayes v3.0 (Ronquist \& Hueselsenbeck 2003) bajo un modelo simple para $28 \mathrm{~S}$ y COI y un modelo mixto para 28S-COI con métodos Monte Carlo de Cadenas de Markov (BMCMC). Se utilizó el protocolo de Wiens y Penkrot (2002) para comprobar los límites específicos entre las secuencias de ADN de E. andrei y $E$. 
fetida. Este método se basa en un diseño del muestreo que incluye: (a) especies focales y no focales para aplicar el test de exclusividad (todos los miembros de un grupo comparten un antecesor común más reciente entre ellos que no comparten con ningún miembro de otro grupo) a las especies focales y b) al menos dos individuos por localidad para hacer las inferencias de flujo genético entre poblaciones. Se necesita una filogenia de haplotipos (o individuos) con designación taxonómica y localidad conocida. Una topología que no agrupe los haplotipos de una localidad dada como clado se tomará como evidencia de flujo genético potencial entre poblaciones. El método se implementó utilizando claves de flujo dicotómico que llevan a varias alternativas para tomar decisiones a nivel de especie.

\section{RESULTADOS}

No hubo diferencias significativas en la producción de capullos de E. fetida en los cuatro cruces experimentales, uno con E. fetida y tres con E. andrei (Fig. 1A; MLG, $\left.\mathrm{F}_{3,20}=0,26, P>0,5\right)$, pero sí hubo diferencias significativas en la viabilidad de los capullos (MLG, $\mathrm{F}_{3,20}=24,03, P>0,0001$ ), de manera que $E$. fetida sólo produjo capullos viables en los cruces intraespecíficos, es decir, no produjo capullos viables en los cruces con E. andrei (Fig. 1B). La producción de capullos de E. andrei fue la misma en las dos poblaciones estudiadas (Vigo y Madrid) (Fig. 2A; MLG, $\mathrm{F}_{1,32}=1,18, P$ = 0,29); y tampoco hubo efecto de los cruces (cuando se cruzó con E. fetida, con $E$. andrei de Vigo y con $E$. andrei de Madrid) (Fig. 2A; $M L G, F_{2,32}=2,26, P=0,12$ ). La población de $E$. andrei de Vigo produjo significativamente menos capullos cuando se cruzó con E. fetida que en los cruces intrapoblacionales $(t=2,34 \mathrm{~g} .1 .=11 P=0,039)$. Sin embargo, la interacción entre población y cruce no fue significativa (población x cruce; MLG, $\left.\mathrm{F}_{2,32}=2,01, P=0,15\right)$. En $E$. andrei, sólo los cruces intraespecíficos produjeron capullos viables, y así la viabilidad de los capullos fue significativamente diferente dependiendo del cruce (Fig. $2 \mathrm{~B}$; $M L G, \mathrm{~F}_{2,34}=41,08, P>0,0001$ ) pero no de la población (Fig. 2B; MLG, $\mathrm{F}_{1,36}=2,08, \mathrm{P}=0,16$ ). La interacción entre población y cruce no fue significativa (MLG, $\mathrm{F}_{2,32}=1,21, P=0,31$ ).

El número de juveniles por capullo fue significativamente más alto en $E$. andrei $(2,75 \pm 0,13)$ que en $E$. fetida $(2,11 \pm 0,24)$. El número total de descendientes producidos por las dos poblaciones de $E$. andrei fue similar y tampoco se observaron diferencias significativas en los cruces entre poblaciones distintas $(\mathrm{P}>0,05$ en todos lo casos).

Con respecto a la delimitación filogenética de E. fetida y E. andrei utilizando secuencias de ADN, las filogenias obtenidas para cada gen por separado no fueron contradictorias y en consecuencia se pudo recurrir a un análisis filogenético combinando las secuencias de los dos genes (Fig. 3). 
A
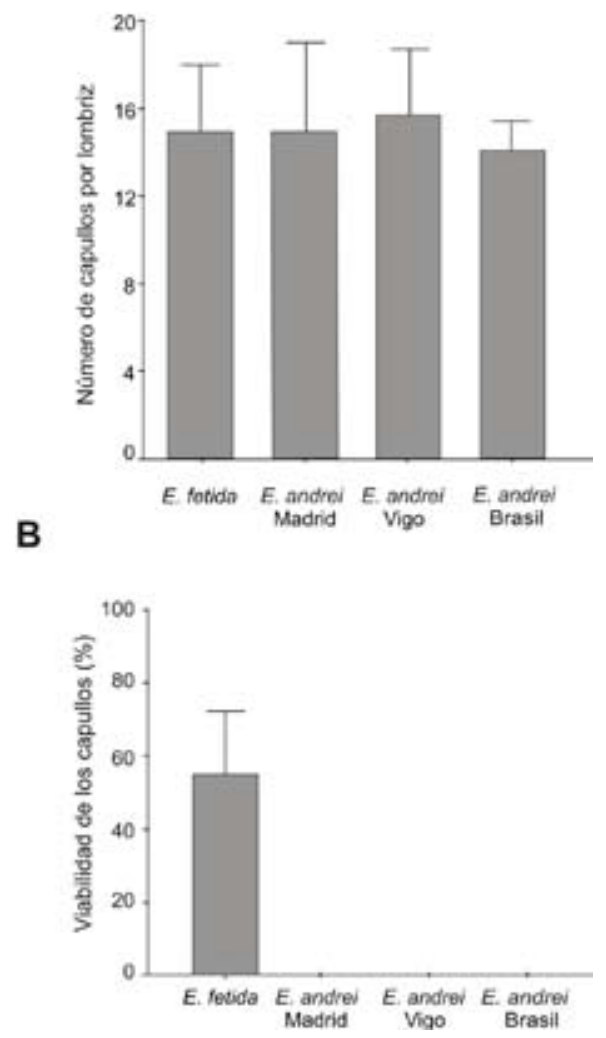

Figura 1. (A) Número de capullos producidos por Eisenia fetida durante 15 semanas y (B) viabilidad de los mismos, después de cruzarse con E. fetida y tres poblaciones de E. andrei. Los valores son la media \pm el error estándar. Véase que no se obtuvieron capullos fértiles en los cruces con $E$. andrei.

En todos los análisis filogenéticos de máxima parsimonia, máxima verosimilitud y bayesiano (BMCMC) de las secuencias de los genes 28S y COI, cada especie de Eisenia apareció como monofilética, aunque el soporte de los clados fue distinto para los dos genes. Los análisis filogenéticos de las secuencias combinadas (28S-COI) mostraron el mismo patrón topológico, es decir que las tres especies de Eisenia aparecen como monofiléticas, pero en esta ocasión el soporte de los clados fue mayor, con una probabilidad de bootstrap elevada $(>90 \%)$ y una probabilidad posterior de 1,0 (Fig. 3). Las relaciones intraespecíficas no se resolvieron bien en ningún caso excepto para E. fetida de Irlanda (EfIr), que aparece claramente separada de las otras dos poblaciones de E. fetida tanto en el árbol combinado (Fig. 3) como en los dos individuales para cada gen (datos no mostrados, véase Pérez-Losada et al. 2005). 
A
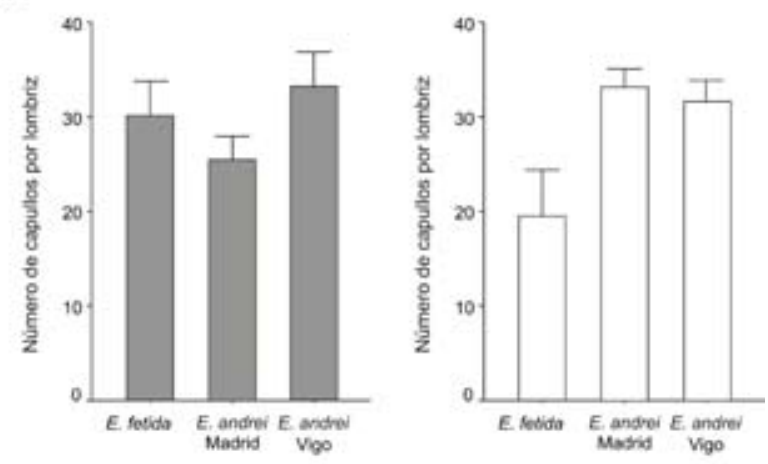

B
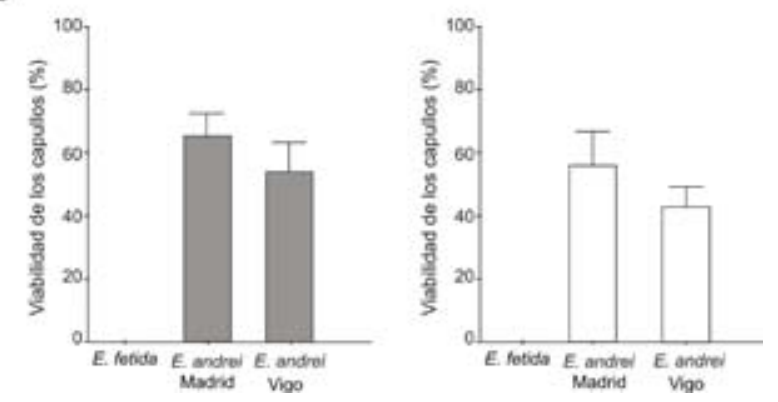

Figura 2. (A) Número de capullos producidos por dos poblaciones de Eisenia andrei (Madrid: barras negras y Vigo: barras blancas) durante 15 semanas en los cruces con E. fetida y con las dos poblaciones de E. andrei. (B) Viabilidad de los capullos de las dos poblaciones de E. andrei (Madrid

(barras negras) y Vigo (barras grises)) en los cruces con $E$. fetida y con las dos poblaciones de $E$. andrei. Los valores son la media \pm el error estándar. Véase que no se obtuvieron capullos fértiles en los cruces con E. fetida.

\section{DISCUSIÓN}

Los experimentos de cruces interespecíficos mostraron que $E$. fetida y $E$. andrei están aisladas reproductivamente porque no hubo descendencia viable de los mismos, de forma que pueden considerarse especies diferentes de acuerdo con la definición biológica de especie (Mayr 1940), corroborando la hipótesis propuesta por Jaenike (1982). No se obtuvo por tanto ningún individuo híbrido. En este sentido, estos resultados contrastan con André (1963), donde la fusión de gametos masculinos de $E$. andrei con gametos femeninos de $E$. fetida produjo híbridos con patrón de pigmentación intermedio, y con Sheepard (1998) que obtuvo también híbridos en cruces interespecíficos, si bien la fertilidad de los capullos en estos cruces fue baja. En consecuencia, nuestros resultados demuestran que la idea de la existencia de una única 


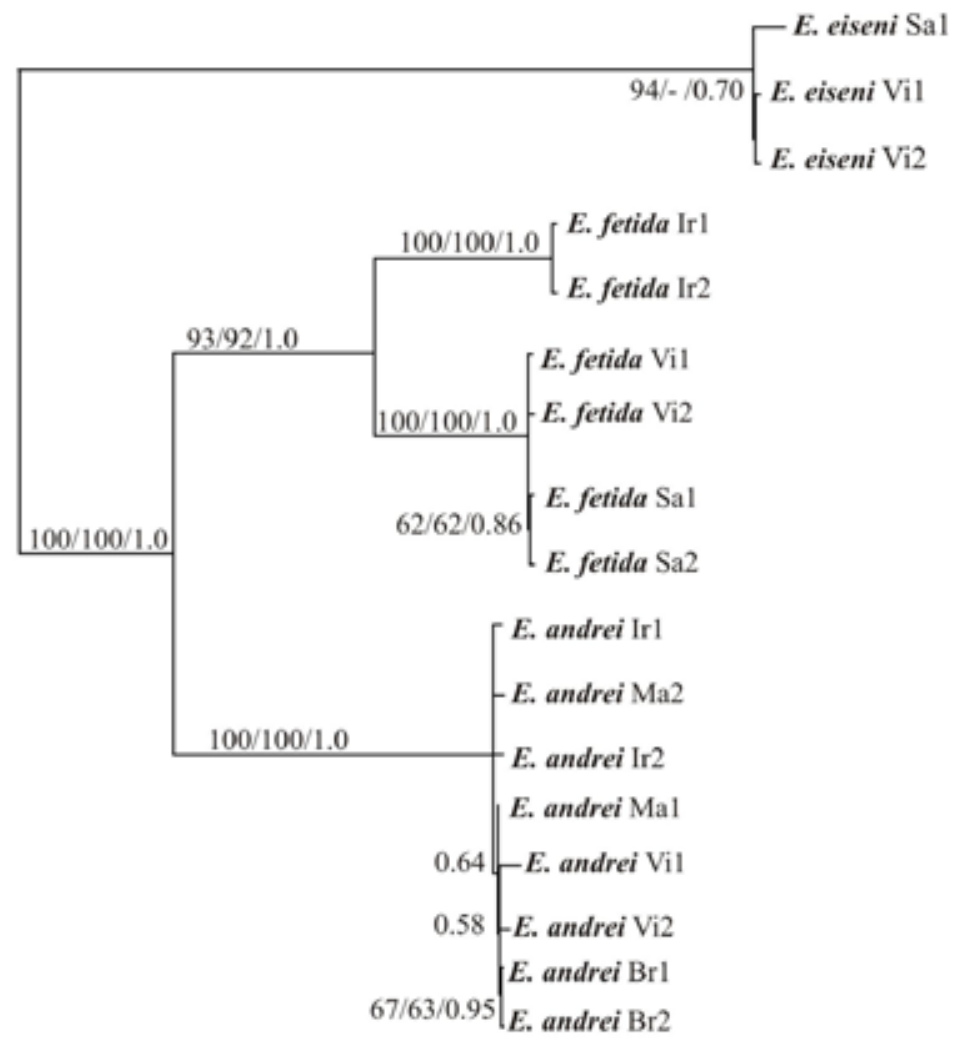

\subsection{5 substituciones/sitio}

Figura 3. Árbol filogenético Bayesiano para 28S-COI. El soporte del bootstrap estimado por MP y ML y la probabilidad posterior se muestran respectivamente sobre las ramas. $\mathrm{Br}=\mathrm{Brasil}, \mathrm{Ir}=\mathrm{Irlanda}$, $\mathrm{Vi}=$ Vigo, $\mathrm{Sa}=$ Santiago, $\mathrm{Ma}=$ Madrid.

especie polimórfica de E. fetida debe rechazarse y sugerimos que, dado que los dos fenotipos se pueden distinguir fácilmente, se puede aplicar el estatus de "buena especie" (Mallet 1995) a los taxones estudiados. Además, nuestros resultados indican que el aislamiento reproductivo entre $E$. andrei y E. fetida es postcópula, probablemente postzigótico, sin que exista un mecanismo eficiente para evitar las cópulas interespecíficas. De hecho, el número de capullos producidos fue similar en los cruces inter e intra específicos de las dos especies, revelando que no existen mecanismos de prevención de la cópula y de la producción de capullos.

Los mecanismos de aislamiento postcópula y postzigóticos impiden el desarrollo del zigoto formando híbridos que no sobreviven, o bien dando lugar a híbridos incapaces de reproducirse. En nuestro estudio se obtuvieron híbridos no viables; por 
tanto, el aislamiento postzigótico en E. fetida y E. andrei se podría caracterizar como intrínseco, dado que depende de problemas en el desarrollo que son relativamente independientes del medio ambiente (Turelli et al. 2001). Parece claro que el aislamiento de ambas especies es incipiente, dado que tiene un profundo efecto en la eficacia biológica de los individuos; ambas especies copulan y producen capullos, aunque éstos son estériles y cabría esperar la aparición de mecanismos que impidan el apareamiento y la producción de capullos con el fin de evitar costes de energía y tiempo innecesarios. De hecho, en nuestro experimento la población de E. andrei de Vigo produjo significativamente menos capullos cuando se cruzó con E. fetida que en los cruces intrapoblacionales, lo que sugiere que la población de $E$. andrei de Vigo invierte menos en capullos híbridos debido a que no son viables. Esto podría ser indicativo del desarrollo de algún tipo de mecanismo prezigótico que evita la inversión de recursos en apareamientos estériles. Además las dos poblaciones de $E$. andrei tuvieron una producción de capullos distinta cuando se cruzaron con E. fetida; esto podría deberse a las diferencias ecológicas entre las dos poblaciones, ya que la población de Madrid proviene de instalaciones comerciales mientras que la población de Vigo procede de una pila de estiércol "natural" y por tanto, pudo estar en contacto con individuos de otras especies. A modo de ejemplo, las hembras del complejo hibridogénico Rana lessonae/Rana esculenta cambian su comportamiento con relación al número de huevos que ponen cuando son amplexadas por machos "indeseados" (Reyer et al. 1999).

En cuanto a la delimitación filogenética de $E$. andrei y $E$. fetida, todos los árboles mostraron que los haplotipos de las especies focales $E$. andrei y E. fetida son exclusivos y que no hay flujo génico entre sus líneas basales. Por tanto se puede concluir que, según el método de delimitación de especies de Wiens y Penkrot (2002), E. andrei y E. fetida son especies filogenéticas diferentes. Estos resultados apoyan estudios genéticos previos basados en alozimas, que indicaron que ambas especies podrían ser diferentes (Jaenike 1982, Henry 1999, McElroy \& Dhile 2001). Adicionalmente, esto concuerda con otros estudios que encontraron diferentes particularidades bioquímicas del factor antibacteriano (Roch et al. 1980), de la actividad bacteriostática de las células secretoras cloragógenas (Valembois et al. 1982) y "marcadores fluorescentes" (fluorescent fingerprints) (Albani et al. 2003) entre las dos especies. En conclusión, según el concepto biológico y filogenético de especie (sensu Mayr 1942) E. andrei y $E$. fetida son dos especies diferentes y por lo tanto las futuras investigaciones en micro o macroevolución, biogeografía, ecología, conservación y aspectos más aplicados (por ejemplo, vermicultura, vermicompostaje y ecotoxicología) con estos dos taxones, deberían ser conscientes de su status específico y de sus implicaciones correspondientes. Por ejemplo, en vermicultura y vermicompostaje se recomienda el uso de E. andrei porque sus tasas de crecimiento y reproducción son más elevadas. En estudios de ecotoxicología, aunque ambas especies tienen características ecoló- 
gicas y probablemente fisiológicas bastante similares, no se puede asumir que los contaminantes tengan el mismo efecto en las dos especies, dado que sus repuestas a factores de estrés pueden ser diferentes. La existencia de un aislamiento postcópula y no precópula en poblaciones simpátricas afecta claramente a la dinámica de poblaciones reduciendo la eficacia biológica individual. Todos estos motivos demuestran que es importante considerarlas como dos especies diferentes y evitar en la medida de lo posible los cultivos mixtos.

\section{AGRADECIMIENTOS}

Este trabajo fue subvencionado por el Ministerio de Educación y Ciencia de España (CGL2006-11928).

\section{LITERATURA CITADA}

Albani, J. R., S. Demuynck, F. Grumiaux \& A. Lepretre. 2003. Fluorescence fingerprints of Eisenia fetida and Eisenia andrei. Photochemistry and Photobiology. 78: 599-602.

André, F. 1963. Contribution a l'analyse expérimentale de la reproduction des lombriciens. Bulletin Biologique de la France et de la Belgique. 97: 1-101.

Bouché, M. 1972. Lombriciens de France. Ecologie et systématique. Annales de Zoologie et Ecologie Animale, Numéro hors-série. Institut National de la Recherche Agronomique, Paris.

Crandall, K. A. \& J. F. Fitzpatrick Jr. 1996. Crayfish molecular systematics: using a combination of procedures to estimate phylogeny. Systematic Biology. 45: 1-26.

Domínguez, J. 2004. State of the art and new perspectives on vermicomposting research. Pp. 401-424. In: C. A. Edwards (Ed.). Earthworm ecology. CRC Press, Boca Raton.

Domínguez, J., A. Ferreiro \& A. Velando. 2005. Are Eisenia fetida (Savigny, 1826) and Eisenia andrei Bouché, 1972 (Oligochaeta, Lumbricidae) different biological species? Pedobiologia. 49: 81-87.

Elvira, C., J. Domínguez, \& M. J. Briones. 1996. Growth and reproduction of Eisenia andrei and E. fetida (Oligochaeta, Lumbricidae) in different organic residues. Pedobiologia. 40: 377-384.

Felsenstein, J. 1985. Confidence limits on phylogenies: an approach using the bootstrap. Evolution. 39: 783-791.

Folmer, O., M. Balck, W. Hoeh, R. Lutz \& R. Vrijenhoek. 1994. DNA primers for amplification of mitochondrial cytochrome c oxidase subunit I from diverse metazoan invertebrates. Molecular Marine Biology and Biotechnology. 3: 294-299.

Henry, W. B. 1999. Differentiation of allozyme loci to distinguish between two species of Eisenia. M.Sc. Thesis, Mississippi State University.

Jaenike, J. 1982. Eisenia foetida is two biological species. Megadrilogica. 4:6-8.

Mallet, J. 1995. A species definition for the modern synthesis. Trends in Ecology and Evolution. 10:294-299.

Mayr, E. 1940. Speciation phenomena in birds. American Naturalist. 74: 249.

Mayr, E. 1942. Systematics and the origin of species. Columbia University Press, New York.

McCullagh, P. \& J. A. Nelder. 1989. General linear models. Chapman \& Hall, London.

McElroy, T. C. \& W. J. Diehl. 2001. Heterosis in two closely related species of earthworm (Eisenia fetida and E. andrei). Heredity. 87: 598-608. 
Pérez-Losada, M., J. Eiroa, S. Mato \& J. Domínguez. 2005. Phylogenetic species delimitation of the earthworms Eisenia fetida (Savigny, 1826) and Eisenia andrei Bouché, 1972 (Oligochaeta, Lumbricidae) based on mitochondrial and nuclear DNA genes. Pedobiologia. 49: 317-324.

Posada, D. \& K. A. Crandall. 1998. Modeltest: testing the model of DNA substitution. Bioinformatics. 14: 817-818.

Pop, A. A., M. Wink \& V. V. Pop. 2003. Use of 18S, 16S rDNA and cytochrome c oxidase sequences in earthworm taxonomy (Oligochaeta, Lumbricidae). Pedobiologia. 47: 428-433.

Reinecke, A. J. \& S. A. Viljoen. 1991. A comparison of the biology of Eisenia fetida and Eisenia andrei (Oligochaeta). Biology and Fertility of Soils. 11: 295-300.

Reyer, H. U., G. Frei \& C. Som. 1999. Cryptic female choice: Frogs reduce clutch size when amplexed by undesired males. Proceedings of the Royal Society of London B. 266: 2101-2107.

Roch, P., P. Valembois \& M. Lassegues. 1980. Biochemical particulars of the antibacterial factor of the two subspecies Eisenia fetida fetida and Eisenia fetida andrei. American Zoologist. 20: 790-794.

Ronquist, F. \& J. P. Huelsenbeck. 2003. MrBayes 3: Bayesian phylogenetic inference under mixed models. Bioinformatics. 19: 1572-1574.

Sheppard, P. S. 1988. Specific differences in cocoon and hatchling production in Eisenia fetida and $E$. andrei. Pp. 83-92. In: C. A. Edwards and E. F. Neuhauser (Eds.). Earthworms in waste and environmental management. SPB, The Hague.

Sims, R. W. \& B. M. Gerard. 1985. Earthworms. In: D. M. Kermack and R. S. K. Barnes (Eds.). Synopses of the British Fauna (New Series), No. 31. Linnean Society, London.

Swofford, D. L. 2002. PAUP*: Phylogenetic Analysis Using Parsimony (* and other methods), version 4.0b8. Sinauer Associates, Sunderland.

Thompson, J. D., T. J. Gibson, F. Plewniak, F. Jeanmougin \& D. G. Higgins. 1997. The ClustalX windows interface: flexible strategies for multiple sequence alignment aided by quality analysis tools. Nucleic Acids Research. 24: 4876-4882.

Turelli, M., N. H. Barto \& J. A. Coyne. 2001. Theory and speciation. Trends in Ecology and Evolution. 16: 330-343.

Valembois, P., P. Roch, M. Lassegues \& N. Davant. 1982. Bacteriostatic activity of a chloragogen cell secretion. Pedobiologia. 24: 191-197.

Wedderburn, R. W. M. 1974. Quasilikelyhood functions generalized linear models and the GaussNewton method. Biometrika. 61: 439-447.

Whiting, M. F. 2001. Mecoptera is paraphyletic: multiple genes and phylogeny of Mecoptera and Siphonaptera. Zoologica Scripta. 31: 93-104.

Wiens, J. J. 1998. Combining data sets with different phylogenetic histories. Systematic Biology. 47: 568-581.

Wiens, J. J. \& T. A. Penkrot. 2002. Delimiting species using DNA and morphological variation and discordant species limits in spiny lizards (Sceloporus). Systematic Biology. 51: 69-91. 